\title{
Fibroma ovárico con células estromales en anillo de sello: un tumor extremadamente infrecuente. Diagnóstico diferencial del tumor de Krukenberg
}

\author{
Luis Contreras M. ${ }^{1}$, Marcela Moreno S. ${ }^{1}$, Tulio Rodríguez A. ${ }^{2}$, Paolo Ricci A. ${ }^{2}$, \\ María Soledad Contreras S. ${ }^{a}$ \\ ${ }^{1}$ Servicio de Anatomía Patológica, ${ }^{2}$ Departamento de Obstetricia y Ginecología, Clínica Las Condes. Santiago, Chile.

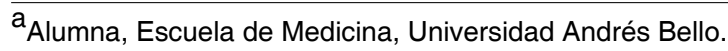

\section{RESUMEN}

La presencia de "células en anillo de sello" en el tejido ovárico es el marcador histológico clásico del tumor de Krukenberg. Un adenocarcinoma metastásico altamente agresivo y de baja sobrevida. En cambio, los fibromas ováricos son tumores del estroma generalmente benignos. Presentamos un caso muy infrecuente de fibroma celular con presencia de células en anillo de sello y revisamos los criterios para el diagnóstico diferencial con el tumor de Krukenberg.

\section{PALABRAS CLAVES: Tumor de ovario, células estromales en anillo de sello, fibroma ovárico, tumor de Krukenberg}

\section{SUMMARY}

The presence of signet-ring cells in ovarian tissue is classically described as histological marker of Krukenberg tumor. It is highly aggressive metastatic adenocarcinoma with low survival. In contrast, ovarian fibroid is a stromal tumor usually benign. We present a very rare case of cellular fibroma with presence of signet-ring cells and we review the criteria for differential diagnosis of Krukenberg tumor.

KEY WORDS: Ovarian tumor, stromal signet-ring cells, ovarian fibroma, Krukenberg tumor

\section{INTRODUCCIÓN}

La presencia de las llamadas "células en anillo de sello" en el tejido ovárico es el marcador histológico clásicamente descrito en el tumor de Krukenberg. Se trata de una metástasis de un adenocarcinoma de origen gastrointestinal o mamario, que se caracteriza por la presencia de mucina intracitoplasmática. Es una neoplasia altamente agresiva y de baja sobrevida (1). A diferencia de lo anterior, los fibromas ováricos son tumores del estroma, compuestos por tejido fibroso productor de colágeno y de conducta generalmente benigna (2).

Presentamos un caso muy infrecuente, de fibroma ovárico con presencia de células en anillo de sello, y revisamos los criterios para un diagnóstico diferencial con el tumor de Krukenberg. 


\section{Caso clínico}

Mujer de 28 años, sin antecedentes mórbidos de importancia, que en un control ginecológico de rutina, se le diagnosticó un tumor ovárico derecho, de carácter sólido, de $3 \mathrm{~cm}$ de diámetro. Se realizó anexectomía con biopsia y estudio contemporáneo, que fue informado como fibroma celular. El tumor correspondió a una formación nodular bien delimitada, sólida, de color pardo amarillenta, de $3 \mathrm{~cm}$ de diámetro mayor, confinada exclusivamente al ovario, sin compromiso de la superficie de éste, ni adherencias a otros órganos (Figura 1A). La exploración macroscópica de la cavidad peritoneal y de la superficie de los órganos pélvicos demostró indemnidad. La paciente evolucionó asintomática y completó un seguimiento y control de 3 años, sin evidencias de recidiva ni compromiso de otros órganos.

Se realizó un estudio y análisis diferido, para conteo mitótico y búsqueda de focos de atipia celular. El espécimen fue fijado en formalina tamponada al $10 \%$, y se tomaron cortes representativos para estudio con Hematoxilina-Eosina, y técnicas histoquímicas e inmunohistoquímicas.

Hallazgos microscópicos. Se encontró reemplazo del parénquima ovárico por una formación nodular relativamente bien delimitada, constituida por células fusadas, monótonas, sin pleomorfismo nuclear, que se disponían en haces arremolinados con áreas hipercelulares (Figura 1B). No se observó necrosis, y se contabilizó hasta una mitosis por diez campos de mayor aumento y escaso colágeno intersticial. En el espesor del tumor se observaron múltiples sectores con presencia de células en anillo de sello, que en conjunto correspondían aproximadamente al $40 \%$ de la masa tumoral. (Figura $1 \mathrm{C}$ y 1D). Estas observaciones hicieron plantear los diagnósticos diferenciales de tumores ováricos con presencia de células en anillo de sello. En el cual se encuentra la descripción característica del tumor de Krukenberg. Para hacer el diagnóstico diferencial, se efectuaron tinciones histoquímicas (Tabla I) con PAS, PAS-diastasa (Figura 2A), azul alcian y mucicarmin, para búsqueda de mucina. Las que resultaron negativas, al igual que las tinciones inmunohistoquímicas para pancitoqueratina (AE1/ AE3) (Figura 2B) y antígeno epitelial de membrana (EMA). Se observó positividad difusa marcada con vimentina (Figura $2 \mathrm{C}$ ) y tinción focal, tanto en las células fusadas como las células anteriormente descritas para calretinina (Figura 2D).

\section{DISCUSIÓN}

El tumor del estroma ovárico con células en anillo de sello es una neoplasia con una frecuencia extremadamente baja (3). En la literatura médica indexada existen 12 casos reportados. En 1976, Ramsy describe el primer caso en una mujer de la misma edad de nuestro reporte (4).
La importancia del hallazgo y confirmación de la presencia de un tumor de estroma ovárico con células en anillo de sello, radica en hacer su diagnóstico diferencial con las metástasis del adenocarcinoma de origen gastrointestinal, también llamado tumor de Krukenberg (5). Las diferencias histológicas y de inmunohistoquímica, que permiten realizar el diagnóstico diferencial se encuentran resumidas en la Tabla I.

Se han descrito en mujeres con rango de edad entre los 21 y 83 años. Característicamente se trata de un tumor unilateral, pero aunque escasos, existen algunos reportes de compromiso bilateral. Son lesiones confinadas al ovario con tamaños descritos entre 2,5 y $13 \mathrm{~cm}(6)$.

De acuerdo a las publicaciones, todos los casos se encuentran libres de enfermedad, con periodos de seguimiento informados entre 1 a 17 años (7). En el caso que reportamos, la paciente ha permanecido asintomática y sin evidencias de enfermedad, completando un seguimiento de 3 años.

Respecto a la descripción macroscópica, la gran mayoría son de tipo sólidos y de color pardo amarillento. Es decir, en todo similares a otros tipos de tumores estromales, e incluso similar al mismo tumor de Krukenberg. La presencia de hemorragia o necrosis no es de utilidad para diferenciarlos.

Desde el punto de vista del análisis histológico, este tipo de tumores se encuentra constituido por dos poblaciones celulares: A) Células con vacuolas citoplasmáticas y núcleo rechazado hacia la periferia, lo que les confiere el aspecto característico en "anillo de sello". Muy similares a las células en anillo de sello del tumor de Krukenberg, aunque sin formar estructuras glandulares. B) Células fusadas, de aspecto fibroblástico, que en ocasiones pueden contener glóbulos hialinos citoplasmáticos. Generalmente no muestran atipia, aunque se han descrito casos aislados con atipia leve (7). El recuento mitótico es bajo, entre 0 y 3 por 10 campos de aumento mayor (7). No se ha descrito presencia de necrosis, hemorragia, ni invasión linfovascular y/o perineural.

Característicamente en el estudio inmunohistoquímico, las células fusadas del tumor de estroma gonadal con células en anillo de sello, muestran positividad para vimentina, calretinina y actina de músculo liso, entre otros marcadores estromales. A diferencia del tumor metastásico de Krukenberg, en que habitualmente son negativos (8). En cambio, las tinciones para mucina intracitoplasmática como PAS, PAS-D, azul alcian y mucicarmin son negativas, al igual que las tinciones inmunohistoquímicas para marcadores epiteliales. A diferencia del tumor de Krukenberg, en el cual las células tumorales son positivas tanto para mucina como para diferentes tipos de citoqueratinas y EMA, lo que demuestra su origen epitelial (9) (Tabla I). 


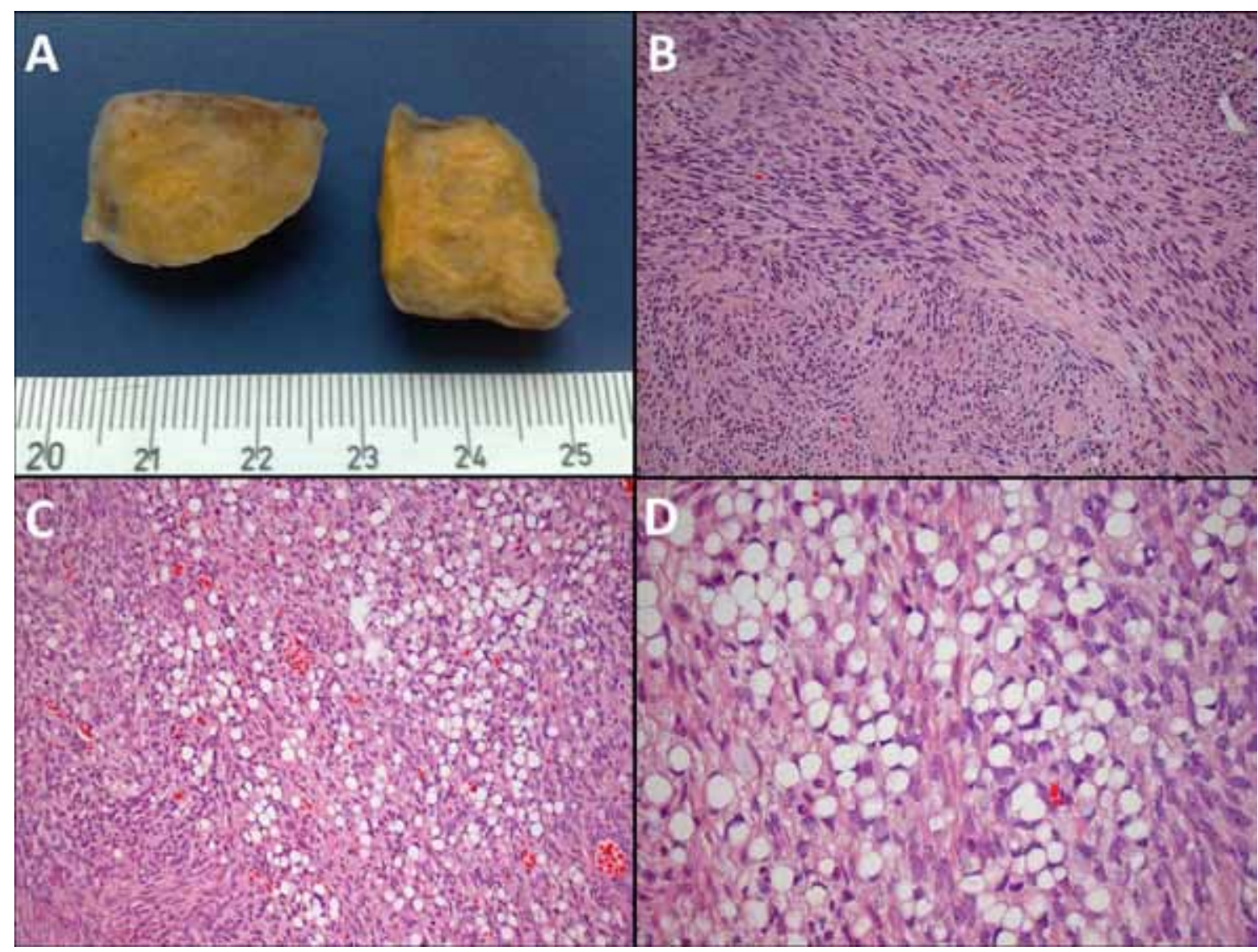

Figura 1. A. Parénquima ovárico pardo amarillo discretamente fascicular. B. Proliferación fuso-celular monótona, sin atipias (HE 100X). C y D. Células con vacuolas intracitoplasmáticas en anillo de sello (HE 200x).

\section{Tabla I}

\section{RESUMEN DE CARACTERÍSTICAS PARA EL DIAGNÓSTICO DIFERENCIAL DE TUMORES OVÁRICOS CON CÉLULAS EN ANILLO DE SELLO}

\section{Característica}

Tumor del estroma gonadal

con células en anillo de sello

Habitualmente unilateral

Negativas

Negativas

Positivas

\section{Lateralidad}

Tinciones para mucina

Tinciones inmunohistoquímicas de estirpe epitelial (pancitoqueratina, EMA)

Tinciones inmunohistoquímicas de estirpe
Metástasis de adenocarcinoma de células en anillo de sello (Tumor de Krukenberg) estromal (vimentina, calrretinina, actina de músculo liso)

\section{Habitualmente bilateral}

Positivas

Positivas

Habitualmente negativas 


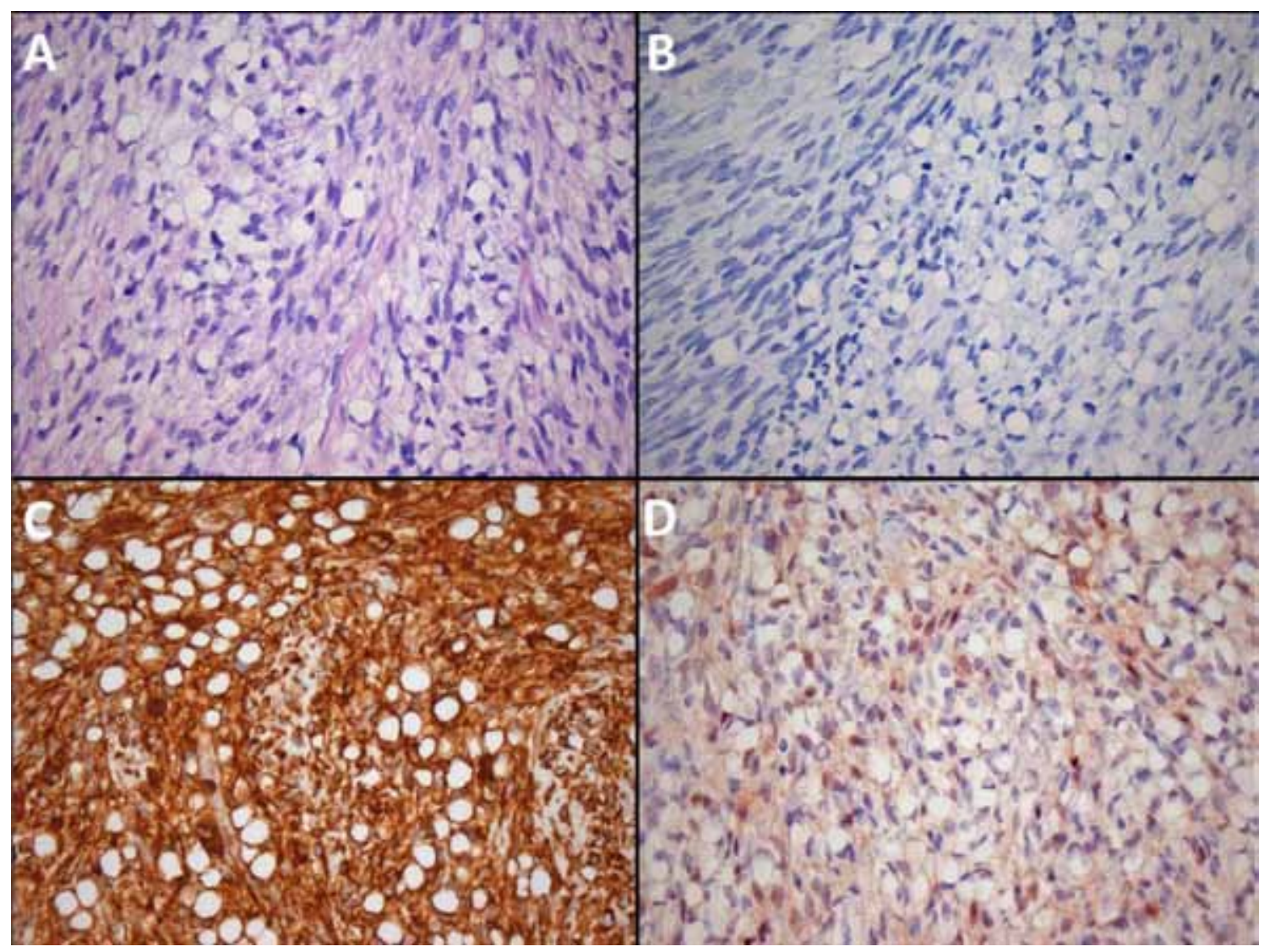

Figura 2. A. Tinción con PAS-diastasa negativa (200X). B. Tinción inmunohistoquímica con pancitoquertina (AE1/AE3) negativa. C. Positividad difusa e intensa para vimentina (200X). D. Positividad focal para calretinina (200X).

Actualmente, las lesiones fibroblásticas ováricas se clasifican como: a) Fibromas típicos: constituidos por células fusadas con abundante colágeno intersticial y escasa celularidad; b) Fibromas celulares: constituidos por células fusadas con escaso colágeno intersticial, sin atipias y con actividad mitótica variable; c) Fibrosarcomas: tumor también de células fusadas, con una tasa mitótica variable, habitualmente mayor a 3 por 10 campos de mayor aumento, y atipia celular significativa. De acuerdo a esta clasificación, el caso descrito corresponde a un fibroma ovárico celular, a pesar de las numerosas células en anillo de sello presentes en la lesión.

Debemos tener presente que los fibromas celulares pueden recurrir, y se ha descrito solo un caso de muerte por este tipo de lesión (10). Por último, debemos mencionar que otro tumor estromal que característicamente presenta células en anillo de sello, es el estromal esclerosante. Sin embargo, la falta de esclerosis en esta lesión descarta inmediatamente este diagnóstico (2).

\section{CONCLUSIÓN}

La presencia de células en anillo de sello en un tumor del estroma ovárico es un hallazgo muy infrecuente, y nos obliga a excluir su etiología metastásica, proveniente de un adenocarcinoma gastrointestinal o tumor de Krukenberg.

Se deben considerar como elementos para el diagnóstico diferencial, los antecedentes clínicos, hallazgos operatorios y el examen histológico. Este último debiera incluir un panel de técnicas de histoquímica como PAS, mucicarmin y azul Alcian. Además de marcadores inmunohistoquímicos epiteliales como EMA y pancitoqueratina, y marcadores estromales como vimentina y calretinina. 


\section{REFERENCIAS}

1. Young RH. From Krukenberg to today: the ever present problems posed by metastatic tumors in the ovary. Part II. Adv Anat Pathol 2007;14(3):149-77.

2. Irving JA, McCluggage WG. Ovarian spindle cell lesions: a review with emphasis on recent developments and differential diagnosis. Adv Anat Pathol 2007;14(5):305-19.

3. Hardisson D, Regojo RM, Mariño-Enríquez A, Martínez-García M. Signet-ring stromal tumor of the ovary: report of a case and review of the literature. Pathol Oncol Res 2008;14(3):333-6.

4. Ramzy I. Signet-ring stromal tumor of ovary. Histochemical, light, and electron microscopic study. Cancer 1976;38(1):166-72.

5. Kiyokawa T, Young RH, Scully RE. Krukenberg tumors of the ovary: a clinicopathologic analysis of 120 cases with emphasis on their variable pathologic manifestations. Am J Surg Pathol 2006;30(3):277-99.
6. Forde GK, Harrison C, Doss BJ, Forde AE, Carlson JW. Bilateral and multinodular signet-ring stromal tumor of the ovary. Obstet Gynecol 2010;116 Suppl 2:556-8.

7. Vang R, Bagué S, Tavassoli FA, Prat J. Signet-ring stromal tumor of the ovary: clinicopathologic analysis and comparison with Krukenberg tumor. Int J Gynecol Pathol 2004;23(1):45-51.

8. Matsumoto M, Hayashi $\mathrm{Y}$, Ohtsuki $\mathrm{Y}$, Ikegami N, Toi $M$, Iguchi $M$, et al. Signet-ring stromal tumor of the ovary: an immunohistochemical and ultrastructural study with a review of the literature. Med Mol Morpho. 2008;41(3):165-70.

9. Suárez A, Palacios J, Burgos E, Gamallo C. Signetring stromal tumor of the ovary: a histochemical, immunohistochemical and ultrastructural study. Virchows Arch A Pathol Anat Histopathol 1993;422(4):333-6.

10. Prat J, Scully RE. Cellular fibromas and fibrosarcomas of the ovary: a comparative clinicopathologic analysis of seventeen cases. Cancer 1981;47(11):2663-70. 